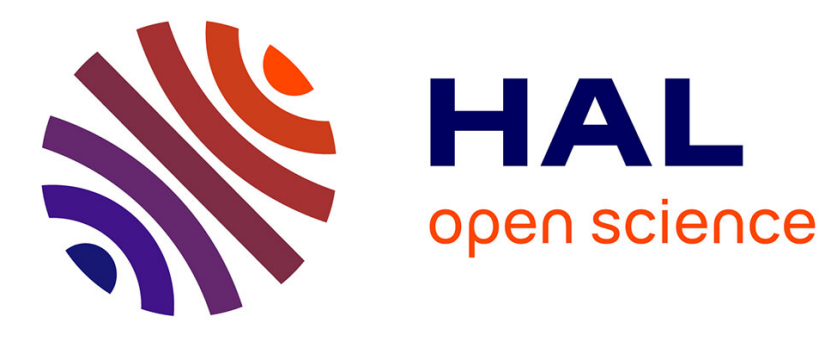

\title{
Nanoplatelets Bridging a Nanotrench: A New Architecture for Photodetectors with Increased Sensitivity
}

Emmanuel Lhuillier, Jean-Francois Dayen, Daniel O. Thomas, Adrien Robin, Bernard Doudin, Benoit Dubertret

\section{To cite this version:}

Emmanuel Lhuillier, Jean-Francois Dayen, Daniel O. Thomas, Adrien Robin, Bernard Doudin, et al.. Nanoplatelets Bridging a Nanotrench: A New Architecture for Photodetectors with Increased Sensitivity. Nano Letters, 2015, 15 (3), pp.1736 - 1742. 10.1021/nl504414g · hal-01438576

\section{HAL Id: hal-01438576 https://hal.science/hal-01438576}

Submitted on 25 Aug 2020

HAL is a multi-disciplinary open access archive for the deposit and dissemination of scientific research documents, whether they are published or not. The documents may come from teaching and research institutions in France or abroad, or from public or private research centers.
L'archive ouverte pluridisciplinaire HAL, est destinée au dépôt et à la diffusion de documents scientifiques de niveau recherche, publiés ou non, émanant des établissements d'enseignement et de recherche français ou étrangers, des laboratoires publics ou privés. 


\section{Nanoplatelets bridging a Nanotrench: a new architecture for photodetectors with increased sensitivity}

Emmanuel Lhuillier ${ }^{1 *}$, Jean-Francois Dayen ${ }^{2 *}$, Daniel O. Thomas ${ }^{3}$, Adrien Robin, ${ }^{1,3}$ Bernard Doudin ${ }^{2}$, Benoit Dubertret $^{3^{*}}$

${ }^{1}$ Nexdot, 10 rue Vauquelin, Paris, France

2 Université de Strasbourg, IPCMS-CMRS UMR 7504, 23 Rue du Loess, 67034 Strasbourg, France

${ }^{3}$ Laboratoire de Physique et d'Etude des Matériaux, ESPCI-ParisTech, PSL Research University,

Sorbonne Université UPMC Univ Paris 06, CNRS, 10 rue Vauquelin 75005 Paris, France.

$\left(^{*}\right)$ Contact: emmanuel.Ihuillier@nexdot.fr, dayen@ipcms.unistra.fr, benoit.dubertret@espci.fr

Abstract: Inter-particle charge hopping severely limits the integration of colloidal nanocrystals films for optoelectronic device applications. We propose here to overcome this problem by using high aspect ratio interconnects made of wide electrodes separated by a few tens of namometers, a distance matching the single nanoplatelet size. The semiconducting $\mathrm{CdSe} / \mathrm{CdS}$ nanoplatelet coupling with such electrodes allows an efficient electron-hole pair dissociation in spite of the large binding energy of the exciton, resulting in optimal photoconductance responsivity. We report the highest responsivity obtained so far for CdSe colloidal material with values reaching $\mathrm{kA} . \mathrm{W}^{-1}$, corresponding to 8 decades of enhancement compared to usual $\mu \mathrm{m}$-scaled architectures. In addition, a decrease of one order of magnitude of the current noise is observed, revealing the reduced influence of the surface traps on transport. The nanotrench geometry provides top access to ion gel electrolyte gating, allowing for a photoresponsive transistor with $10^{4} \mathrm{on} /$ off ratio. A simple analytical model reproduces the device behavior and underlines the key parameters related to its performance.

Keywords: semiconductor nanoparticles, quantum dot, nanoplatelets, photodetection, electrolyte gating, noise

TOC:

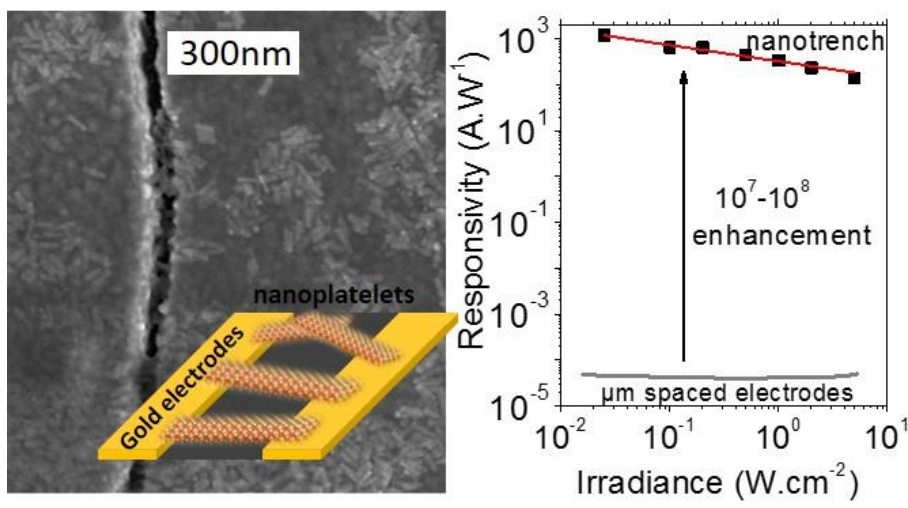




\section{INTRODUCTION}

The implementation of colloidal quantum dots (QD) films in optoelectronic devices ${ }^{1}$ is motivated by the ease of solution processing, their stability in oxygenated atmosphere and the easy process scalability, combined with the unique and tunable quantum nature of semiconductor nanoparticles. While the degree of control of intrinsic optical properties of isolated particles is remarkable, mastering the electrical and optoelectronic properties of a large ensemble of correlated constituents remains challenging. Charge transport in a QD film is a multiscale mechanism, where inter-grain hopping processes occur at the nanoparticle scale, while a percolative regime is predominant at larger scale, being directly influenced by the film morphology (cracks, electrostatic landscape...). Consequently, the interparticle tunnel barrier needs to be tuned and large scale ordering control is mandatory. Surface atomic passivation, such as $\mathrm{S}^{2-2}, \mathrm{SCN}^{-3}$ or $\mathrm{Cl}^{-4}$ and metal chalcogenides ligands ${ }^{5}$, are well-documented techniques capable to shorten and lower the interparticle tunnel barrier, but they generally require the use of polar solvents. This comes at the price of limiting the methods usable to build or self-assemble the QD film, with a resulting limited long-range ordering in samples promoting percolating transport mechanisms detrimental to the device properties. In randomly packed films, the photoactivated carrier must perform a random walk before reaching the source and drain electrodes. This typically involves only a limited fraction of the sample for transferring the charges, with possibly several competing paths possible, both resulting in drastic loss of device performance. At a basic level, the intrinsic electronic properties of the QD get masked by the transport signature of the percolative processes. These drawbacks can be possibly overcome by a strategy taking advantage of hybrid structures, as illustrated by the use of QD-graphene hybrid 6 , where the absorption and hole-pair creation occurs in the QD, and the charge transfer occurs through the graphene intermediate. Another approach, allowing to keep the charge transport signature of the colloids, is the use of electrodes separated by a few $\mathrm{nm}$ only, electrically addressing a single nanoparticle ${ }^{7}$. In this particular situation, the short transport length also reduces the carriers transit time, which positively impacts the photoconductive gain of the device ${ }^{8,9}$. Several methods have been proposed to realize these nanogaps: e-beam lithography ${ }^{10,11}$, self-alignment method ${ }^{12,13}$, electromigration ${ }^{14,15}$, to name a few. Making contact with a single nanoparticle provides unique insight into fundamental understanding and eliminates the complications associated with hopping-assisted percolating mechanism, but suffers from severe drawbacks. The first of these drawbacks is that room-temperature unique quantum properties of the dots imply sizes in the $10 \mathrm{~nm}$ scale, where electrodes fabrication reaches its limits of reliability, even when the most sophisticated lithography techniques are used. Secondly, the non-reproducibility from sample to sample in the electrode/nanoparticle interface requires statistical studies or scientifically-sound samples selection procedures. Finally, the poor effective light absorption due to the reduced active area and the lack of scalability of the proposed fabrication process severely limit the possible realization of devices relevant for applications. All these intrinsic difficulties make the development of new strategies mandatory for reliable and scalable device architectures. Alternatively, better-suited choices for QD geometries could greatly help their device integration. Of particular interest are nanoplatelets (NPL) ${ }^{16,17}$, exhibiting a planar rectangular structure of a few atoms thick, providing a new class of nanomaterial with 2D quantum properties relevant for optoelectronic applications. We recently demonstrated that NPL are interesting candidates for photodetection and that their responsivity can be enhanced by 3 orders of magnitude while used in a phototransistor configuration interfacing several $\mu \mathrm{m}$ long NPL arrays ${ }^{18}$. In spite of these results, the overall response remains low and new strategies need to be developed. Here we propose to use a shadowing angle evaporation approach to create electrical interconnects separated by a short nanometric distance (typically 40-50 nm) over a very wide uniform length (typically $50 \mu \mathrm{m}$ ). ${ }^{19}$ The nanotrenches are ideally suited to electrically address a large ensemble of NPLs connected in parallel, with transport properties occurring over a length scale corresponding to the single particle size. This promotes the desired simplified and more efficient charge transfer mechanisms, free of hopping and percolation, ${ }^{20,21}$ and at the same time offers a larger and more effective device area for optical detection. 


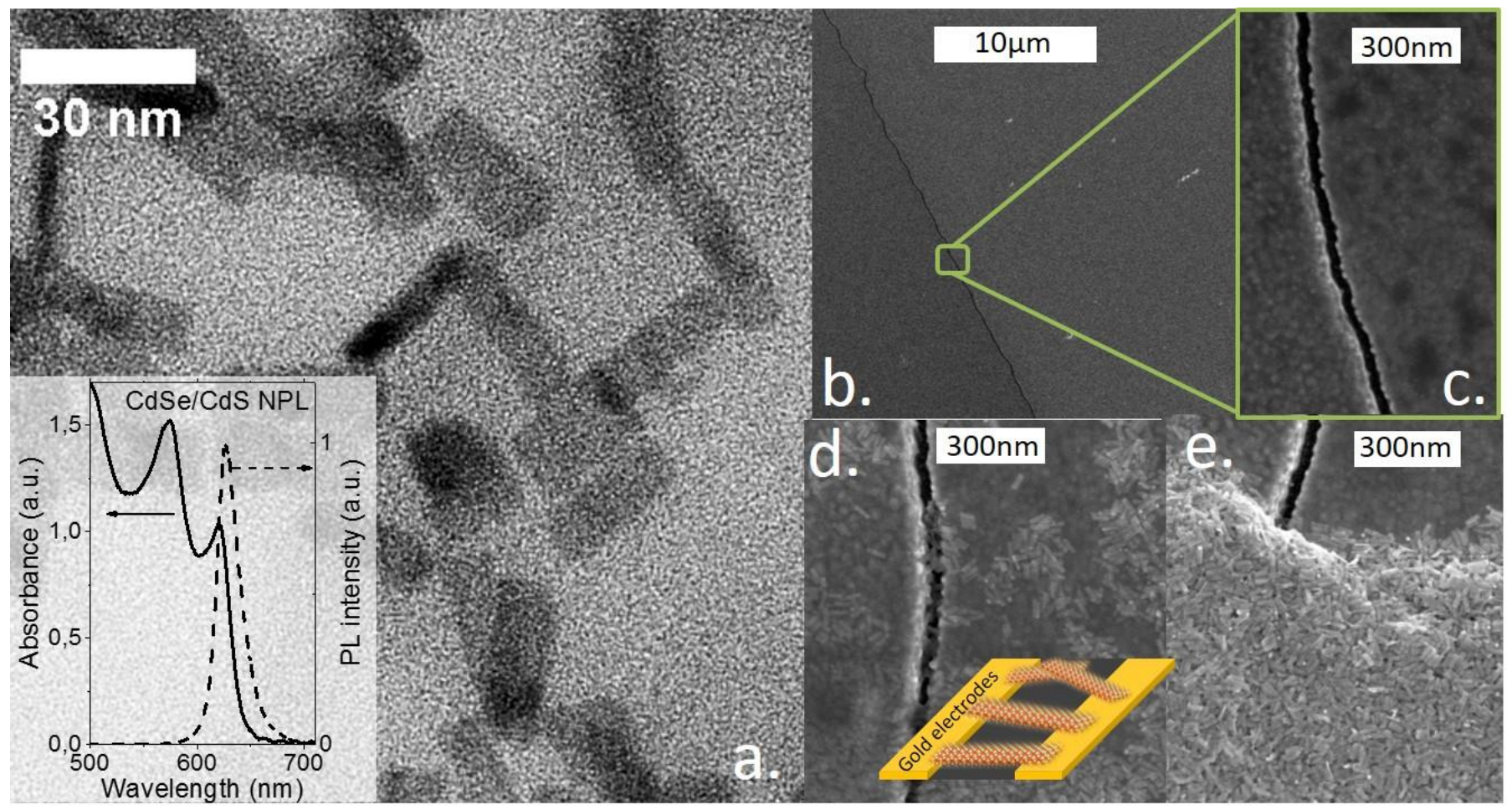

Figure 1 a TEM image of the NPL. The inset showsan Absorption and photoluminescence spectrum for CdSe/CdS NPL. For PL the NPL solution was excited at 350nm by a $150 \mathrm{~W}$ xenon light with a $1 \mathrm{~nm}$ slit. $b$-e are $S E M$ images of naked nanotrenches (b-c) or decorated with low density (d) and high density (e) of NPL, the NPL appears as whitish rectangle. Ond the inset is a scheme of the gold contact connected by the NPL.

We use CdSe/CdS NPL as colloidal nanocrystal material. The CdSe cores, synthetized following the procedure described in reference 17, correspond to a $2 \mathrm{D}$ sheet of $1.5 \mathrm{~nm}$ thick with $10 \mathrm{~nm} \times 40 \mathrm{~nm}$ lateral extension, (Figure 1a). We further grow a CdS shell using the C-ALD procedure, as proposed in 22. The NPL have a first excitonic feature at $630 \mathrm{~nm}(2 \mathrm{eV})$ attributed to the heavy hole-conduction band transition, while the second transition feature at $575 \mathrm{~nm}$ results from the light hole conduction band transition ${ }^{17}$. See the supporting information for more information on the growth of nanoplatelets. Nanotrenches are fabricated through two steps of optical lithography combined with angle deposition ${ }^{19}$ following a shadow-edge deposition strategy. It allows us to define a 40-50nm gap (see figures S1 and S2 of supplementary material), homogenously reproduced along several tens of $\mu \mathrm{m}$ in between electrodes (up to 50 $\mu \mathrm{m}$ in the present work, see Figure $1 \mathrm{~b}$-e and figure S2). The NPL capped by $\mathrm{S}^{2-}$ ligands ${ }^{2}$ are then drop casted onto the nanotrench and annealed at $100^{\circ} \mathrm{C}$ to evaporate the solvent. Final devices are coated with a $200-300 \mathrm{~nm}$ thick film of NPLS.

One of the key advantage of the nanotrench architecture is the possibility to apply fairly large electric field $(F)$ over the structure $\left(F>100 \mathrm{kV} . \mathrm{cm}^{-1}\right)$. It makes it possible to measure a current under dark condition while no signal (i.e. below the setup resolution) was obtained when using standard $\mu \mathrm{m}$-spaced electrodes. A scheme of the experimental setup is given on figure $\mathrm{S} 3 \mathrm{a}$ and $\mathrm{b}$. The signal is also strongly modulated while under illumination, see Figure $2 \mathrm{a}$, with about 5 decades modulation with 50nW $\left(\approx 5 \mathrm{~W} . \mathrm{cm}^{-2}\right)$ illumination only. The photoresponse is fast with a bandwidth of $3 \mathrm{kHz}$, see Figure $2 \mathrm{~b}$ and $\mathrm{c}$. The system responsivity $R=\frac{i_{\text {photon }}}{P_{\text {light }}}$ (ratio of the photocurrent $i_{\text {photon }}$ over the incident light into the nanogap $P_{\text {light }}{ }^{23}$ ) can reach the $\mathrm{kA} . \mathrm{W}^{-1}$ order of magnitude, which corresponds to an external quantum efficiency $\eta g=\frac{h v \cdot R}{e}=3000$ electrons/photons (with $h v \approx 3 \mathrm{eV}$ the photon energy and e the proton charge), see Figure $2 \mathrm{~d}$. Photoconduction data observed in the nanotrench geometry reveal an enhancement of almost 8 orders of 
magnitude of the device responsivity, when compared to data obtained using $\mu \mathrm{m}$-spaced electrodes ${ }^{18}$ (showing values in the tens of $\mu \mathrm{A} . \mathrm{W}^{-1}$ range, see Figure $2 \mathrm{~d}$ ) for the same NPL film preparation. A part of this improvement simply results from a geometrical effect, since a shorter channel comes with a short transit time and thus a high gain (defined as the ratio of the exciton lifetime over the transit time). Nevertheless, this effect only contributes for a factor of $\mathbf{2 0 0}$ (ratio of the channel length) to the increase of the responsivity. The additional increase observed can be explained by a combined effect of (i) the absence of cracks of defects in film at the scale of the nanotrench and (ii) an enhanced charge dissociation. Indeed, the measured sensitivity is one decade above the highest value reported for CdSe colloidal material ${ }^{24}$ and compares to the best value obtained for PbS CQD 25 . The high dielectric constant of $\mathrm{PbS}$ makes the exciton far easier to dissociate due to the poor bonding of the electron hole pair. In contrast, in CdSe and in particular in NPL, binding energy as high as $E_{\text {binding }}=250 \mathrm{meV}$ has been reported ${ }^{26,27}$. Such strong coupling of the electron hole pair can contribute to the poor response obtained using $\mu \mathrm{m}$-spaced electrodes. The nanotrench architecture, because of the larger electric field $F_{N P L}$ it can create along the NPL $\left(F_{N P L}>100 \mathrm{kV} . \mathrm{cm}^{-1}\right)$, provides a simple key to unlock this important technological bottleneck. Because of its $\mathrm{nm}$ spaced electrodes, the energy drop per particle $E_{N P L}=e . F . L_{N P L}$ - with $L_{N P L}$ the particle size -can easily overcome the NPL binding energy. This simply explains the significantly more efficient photocarrier separation observed in our devices.
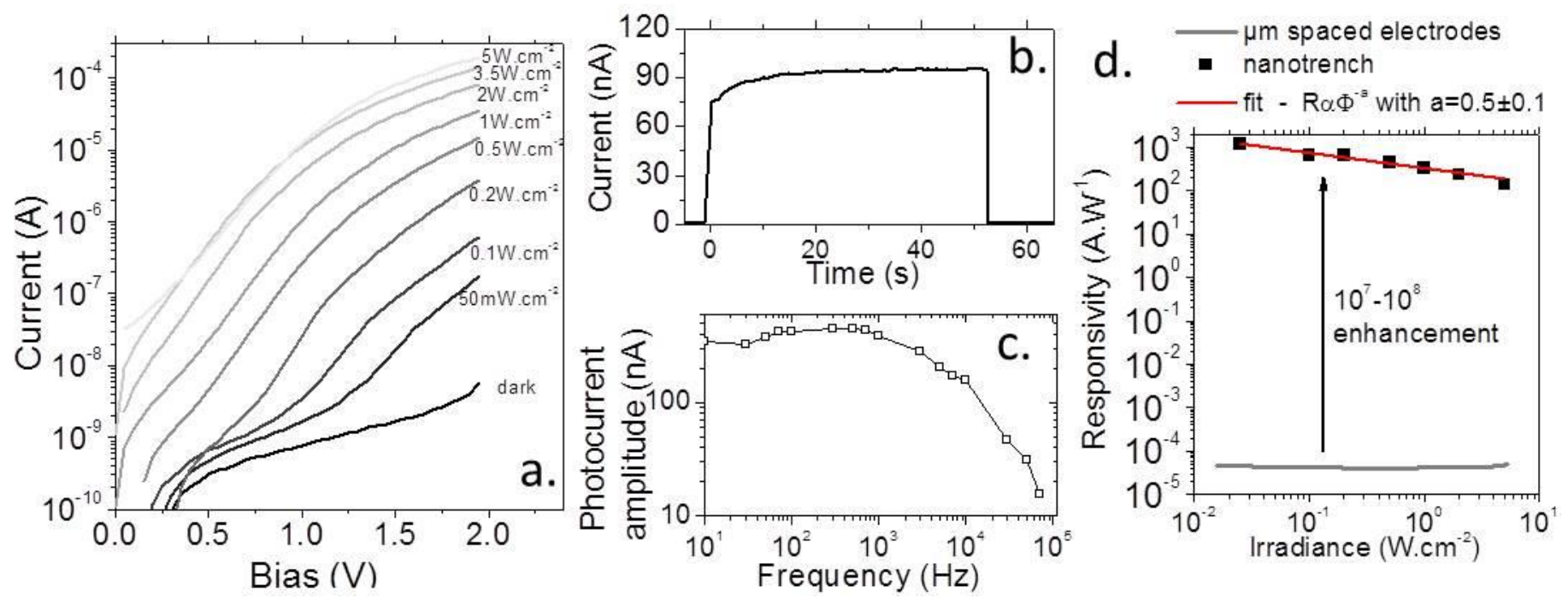

Figure 2a. Current as a function of the drain bias under dark and illuminated condition. $b$. Current as a function of time in response to a light illumination for a film of CdSe/CdS nanoplatelets under $1 \mathrm{~V}$ drain bias and $1 \mathrm{~W} . \mathrm{cm}^{-2}$ light power at 405nm). c Frequency dependence of the photocurrent, measured under $1 \mathrm{~V}$ and $5 \mathrm{~mW} . \mathrm{cm}^{-2}$ light illumination at $405 \mathrm{~nm}$. $d$ Responsivity as a function of the irradiance for a film of CdSe nanoplatelets under a $2 \mathrm{~V}$ bias illuminated by a laser at $405 \mathrm{~nm}$. The laser power varies from $0.25 \mathrm{~mW}$ to $50 \mathrm{~mW}$.

The power dependence of the responsivity exhibits a power law behavior with an exponent equal to -0.5, see Figure $2 \mathrm{~d}$. According to the model described in reference 9, such dependence is typical of band gap recombination-limited devices and result from a higher band-to-band wavefunction overlap induced by the stark effect. This is in contrast to trap-limited systems exhibiting a photoresponse independent of incident power, as observed on Figure $2 \mathrm{~d}$ for the $\mu \mathrm{m}$ spaced electrodes. In the nanotrench geometry, the transport is dominated by paths without traps. This speculation is consistent with the previously observed inhomogeneous photocurrent map over the nanotrench, see reference 8 . The data of Figure $2 d$ therefore strongly supports our hypothesis of charge transfer free of hopping in our devices. 

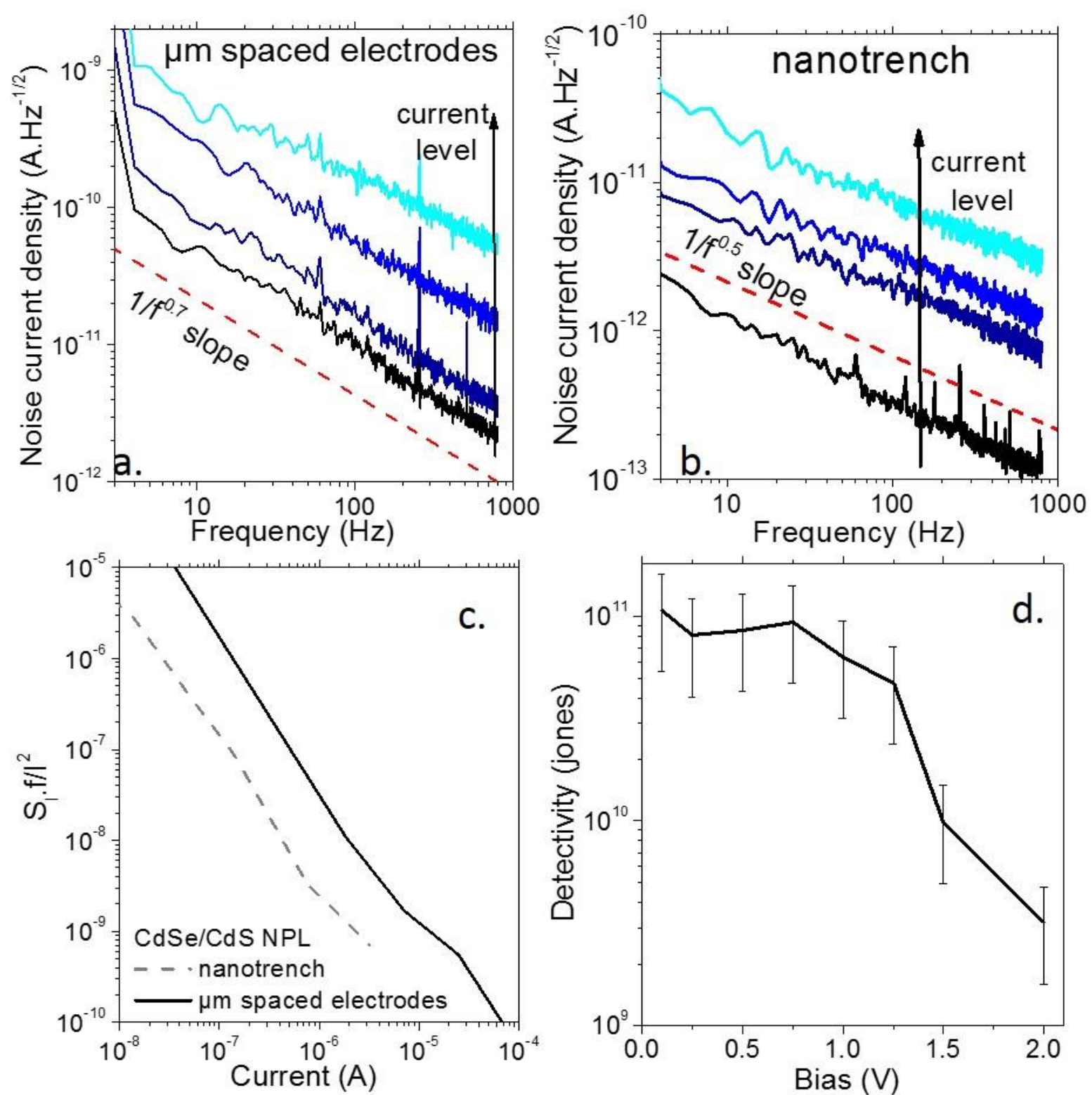

Figure 3 a. Noise as a function of frequency for a CdSe/CdS NPL film under different level of light irradiance connected over $10 \mu \mathrm{m}$ spaced interdigitated electrodes. B. Same as in a. for a 50nm spaced nanotrench. C. Relative noise (noise power density multiplied by the frequency $-100 \mathrm{~Hz}$ - and divided by the square of the current) as a function of the current for a film of CdSe/dS NPL connected over a nanotrench and over $\mu m$ spaced electrodes. $d$. Detectivity of a CdSe/dS NPL film connected over a nanotrench, as a function of bias.

Electronic noise studies provide essential complementary information that validate the use of such devices for photodetection purposes. Noise measurements relate to the detectivity (signal to noise ratio), which is essential for the device performance figure of merit. There is a significant need of data on colloidal based devices, with studies usually limited to assumption that shot noise is prevailing, while detailed quantitative measurement of the noise spectrum is lacking. We are aware of a very few recent studies ${ }^{28,29}$ pointing out that the $1 / f$ type of noise prevails in colloidal based devices, with a magnitude tunable over decades depending on the interparticle couductance ${ }^{28}$. Here we argue that our proposed nanotrench geometry provides unique advantage for reducing the device current noise.

Further confirmation of the predominant intrinsic NPL transport signature is deduced from the current noise reduction, see figure S3 c for details about the experimental setup. The latter relates to the shrinking of the active electrical volume and to the change of transport mechanism when compared to macroscopic samples. Indeed it has been previously shown that hopping transport in a film of colloidal nanocrystals exhibits a strong $1 / f$ noise signature ${ }^{28,29}$. This is related to the mobility fluctuations due to variations of coupling from nanocrystals to nanocrystals. Such significant $1 / f$ noise indeed prevails in our NPL films connected to $\mu \mathrm{m}$ spaced electrodes, see Figure $3 a$. In this case, the current noise density presents a $1 / f^{0.7}$ dependence. Flicker noise is also found on 
nanotrench devices, see Figure $3 b$. However, the relative noise intensity $\left(S_{1} / I^{2}\right)$, with $/$ the current and $S$, the power noise current density is reduced by a decade with the nanotrench architecture, see Figure 3c. Again, in nanotrench architecture the carriers experience less NPL (mainly one single only) and are consequently less sensitive to the change of mobility coupling compared to the long percolating hopping path in $\mu \mathrm{m}$-spaced electrodes. From these noise measurements we are able to evaluate the device detectivity $D^{*}=\frac{R \sqrt{A}}{i_{n}}$ with $R$ the responsivity, $A$ the optical area and $i_{n}$ the noise current density. We obtain value up to $10^{11}$ jones, see Figure $3 \mathrm{~d}$. The detectivity value is approximately constant up to $1 \mathrm{~V}$, and then drops as the current significantly increases because the applied bias over a particle starts to be of the order of half the material band edge energy. The value is currently limited by the small optical area inherent to the simple nanotrench geometry used here. Such limitation can easily be overcome by using several nanotrenches in a parallel geometry.

To further investigate the transport properties of the NPL/nanotrench hybrid, we gate the system using ion-gel electrolyte as proposed in ref 18 and $30 . \mathrm{LiCLO}_{4}$ dissolved in polyethylelne glycol is used as electrolyte and a gold side gate is used to tune the Fermi level of the system. The gating is air operable. As expected for $\mathrm{CdSe} / \mathrm{CdS}$ colloidal material we observe a n-type behavior, with a on/off ratio close to 4 decades, see Figure 4a.

To further understand the mechanism of transport involved in this short channel transistor we modeled the system band structure under the simplest hypothesis. The Poisson equation $\frac{d^{2} V}{d z^{2}}=-\frac{e^{2} N_{D}}{\varepsilon \varepsilon_{0}}$ with $N_{D}$ the doping density, $\varepsilon$ the material dielectric constant, $\varepsilon_{0}$ the vacuum permittivity, leads to a parabolic bending of the band. From this equation we can expect a parabolic expression for the band profile in absence of applied electric field: $V(z)=a z^{2}+b z+c$. The boundary conditions are given at the interface by $V(0)=V(L)=V_{s}$ with $V_{s}$ the interfacial potential and $L$ the electrodes spacing bridged by a NPL. Assuming a Schottky contact we estimate $V_{s}$ to be $V_{s}=\phi_{M}-\chi_{Q D}$ where $\phi_{M}$ is the metal electrodes work function and $\chi_{Q D}$ the QD electron affinity. The latter is itself related to the bulk electron affinity $\chi_{b u l k}$ by $\chi_{Q D}=\chi_{\text {bulk }}-E_{c}$ with $E_{c}$ the confinement energy in the confinement band. A reasonable estimate of $E_{c}$ is $E_{c}=\frac{m_{v}^{*}}{m_{v} *+m_{c} *}\left(E_{Q D}-E_{b u l k}\right)$ where $m_{v} *$ is the valence band effective mass, $m_{c} *$ the conduction band effective mass, $E_{Q D}$ the nanoparticle band edge energy and $E_{b u l k}$ the bulk band gap. The values of the whole set of parameters used for the modelling are given in table S1.

Moreover, we assume that in the middle of the nanotrench the Fermi level is in the middle of the gap (intrinsic behavior) which leads to $V(L / 2)=E_{Q D} / 2+\Delta E_{F}\left(V_{G S}\right)$ with $\Delta E_{F}\left(V_{G S}\right)$ the shift of the Fermi level related to the gating. The use of this expression is reasonable if the depletion length given by $z_{d e p}=\sqrt{\frac{2 \varepsilon \varepsilon_{0} k_{b} T}{e^{2} N_{D}}}$ is significantly shorter than the nanogap size. With ion-gel electrolyte, sheet density of $10^{14} \mathrm{~cm}^{-2}$ are easily achieved ${ }^{31}$, corresponding to $10^{19} \mathrm{~cm}^{-3}$ as doping density for $100 \mathrm{~nm}$ thick film. We can estimate that the depletion length is consequently just a few $\mathrm{nm}$ in our system, which remains a decade shorter than the channel size. We thus finally obtain, taking into account the applied electric field $F$ between the drain source electrodes,

$V(z)=\frac{4}{L^{2}}\left[E_{Q D} / 2+\Delta E_{F}\left(V_{G S}\right)-V_{s}\right]\left(z^{2}-L z\right)+V_{s}+e F z$

The parameters used for the modelling are given in table S1. The Figure $4 \mathrm{~b}$ and figure $\mathrm{S} 5$ shows the obtained energy diagram using equation (1) under different drain and gate bias. Due to the gold electrodes used and the confinement in the NPL, we estimate that the Schottky barrier at the contact is of $0.5 \mathrm{eV}$. In the intrinsic regime the bending of the band clearly undermines the electron injection. 

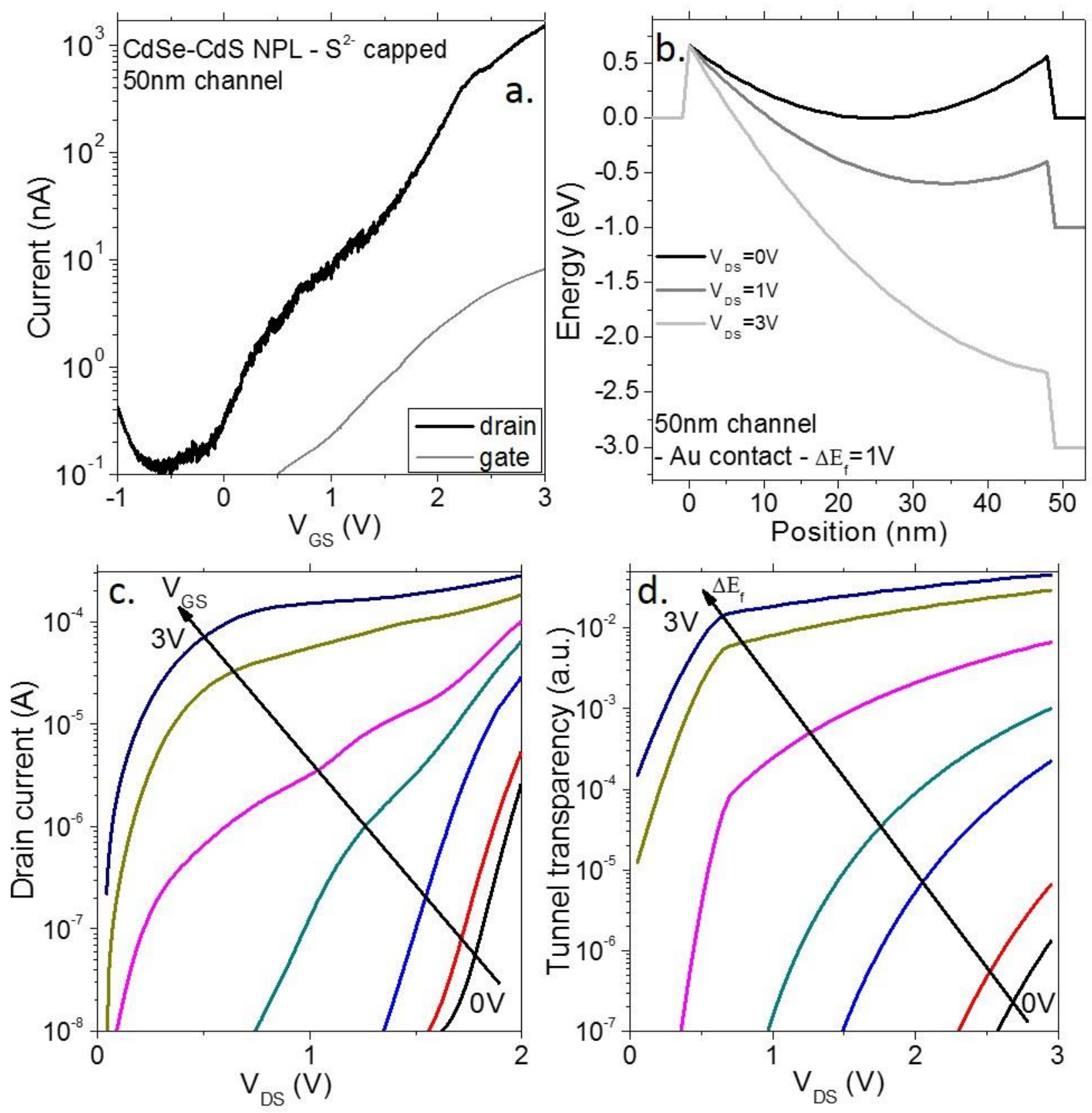

Figure 4 a. Drain current as a function of the gate voltage for a film of $\mathrm{CdSe} / \mathrm{CdS}$ nanoplatelets bridging nanotrench electrodes spaced by a $50 \mathrm{~nm}$ gap. The drain current is set to $0.5 \mathrm{~V}$. b Scheme of the band structure based on equation (1) for a $1 \mathrm{~V}$ displacement of the Fermi level and different drain source bias. $c$. Drain current as a function of the drain voltage for a film of $\mathrm{CdSe} / \mathrm{CdS}$ nanoplatelets, under different gate bias (the step of gate bias is $0.5 \mathrm{~V}$ ). The drain bias is set to $0.5 \mathrm{~V}$. d. Tunnel transparency as a function of the applied bias over the nanotrench for different tunning of the Fermi level.

Then, to relate this band structure to the transport measurement we choose to evaluate the tunnel transparency of the contact. We use the Wentzel-Kramers-brillouin (WKB) approximation, for which the transparency is given by $T(E)=\exp \left(-2 \sqrt{\frac{2 m_{c}^{*}}{\hbar^{2}}} \int_{0}^{L} \sqrt{V_{C}(z)-E} d z\right)$. The transport data (Figure 4c) are compared with the tunnel transparency related to the Schottky contact barrier obtained by numerically integrating the conduction band profile (Figure 4d). This simplest modelling qualitatively reproduces the observed bias and gate dependence of the current.

We now discuss the expected transport mechanism to be promoted according to the tunnel transparency of the contact. At low gate bias, we reproduce the threshold aspect of the current related to the exponential dependence of the transparency with the electric field (introduced on Figure $5 d$, through the threshold gate voltage $V_{t}$ ). In this range of low gate bias, the transport is limited by the carrier injection and current only occurs when the potential 
drop per particle overcomes at least half the energy gap $E_{G}$ (with $E_{G} \approx 2 e V$ ). The associated transport mechanisms are band-to-band tunneling (injection of electron from the valence band to the conduction band) and mid gap states assisted tunneling, see Figure $5 \mathrm{c}$.

At higher gate bias we can distinguish two regimes. Below 0.5V of drain bias (roughly the height of the Schottky barrier) a strong bias dependence indicates that a significant part of the channel contributes to the tunnel barrier. In this range, the shift of the Fermi level may even lead to the formation of an electrostatically induced confined dot in the NPL and transport occurs over two steps of tunneling: one to enter the electrostatically confined area and one to leave it, see Figure 5a. On the other hand, for drain biases above the Schottky barrier, the bias dependence is significantly reduced, see Figure 4c, because the tunnel barrier is now sufficiently thin to ensure a good electron injection from the contact to the conduction band, see Figure 5b. A detailed description of the above-mentioned regimes are out of the scope of this paper, nevertheless it is clear from our modelling that for such nanotrench based devices the contacts are driving the transport properties and need to be efficiently engineered to ensure optimized device performances.

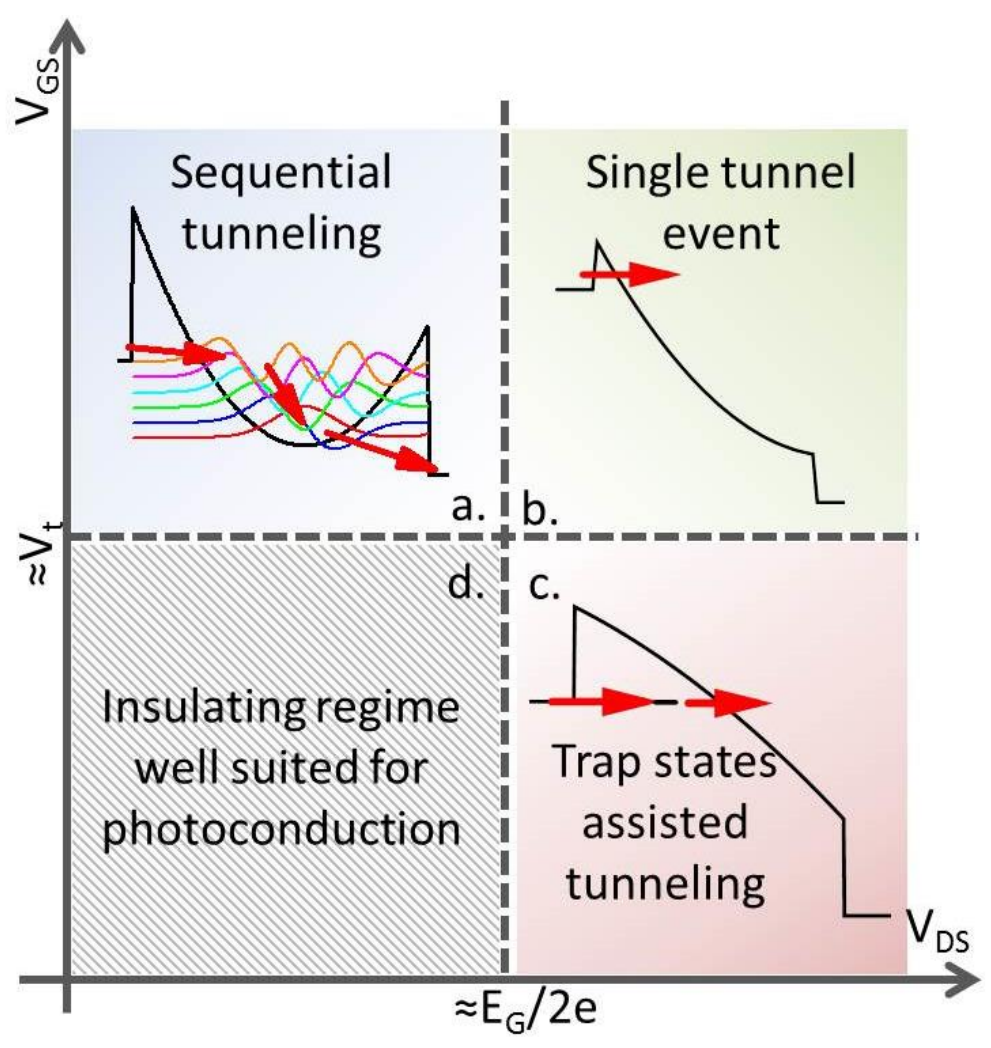

Figure 5 Phase diagram of the different transport processes as a function of drain and gate bias, respect to the energy gap $E_{G}$ of the NPL and the threshold gate voltage $V_{t}$.

\section{CONCLUSION}

We investigate the transport and phototransport properties of colloidal nanoplatelets films connected over a nanotrench with inter-electrodes spacing matching the nanoplatelets length. In this configuration, we obtain significantly improved photodetection properties compared to usual $\mu \mathrm{m}$-spaced electrodes with a photoresponse enhancement reaching 8 decades for a given material. In addition, this geometry reduces the influence of the traps and leads to a relative noise reduced by a decade. We also investigate the dark transport properties of the devices using an ion gel gating procedure. A simple analytical model illustrates the band structure under high electric field and a large shift of the Fermi level, which provides insight into observed I-V curves. Our model highlights the importance of the contact for devices whose channel length matches the contacted nanostructure size. More generally, combining nanoplatelets and nanotrench architecture presents itself as a unique device architecture to 
develop a new family of detectors exploiting the unique properties of the physics of confined carriers in colloidal nanostructures.

\section{Supplementary information}

Additional informations include details about the chemical product and precursors, nanotrench fabrication, device characterization. This material is available free of charge via the Internet at http://pubs.acs.org.

\section{Acknowledgements}

The authors thank Xiang Zhen Xu for her help with TEM imaging. We thank Agence National de la Recherche for funding through grants SNAP and Labex NIE 11-LABX-0058_NIE within the Investissement d'Avenir program ANR-10-IDEX-0002-02. This work has been supported by the Region lle-de-France in the framework of DIM Nano-K.

\section{REFERENCES}

\footnotetext{
${ }^{1}$ Talapin D., Lee J.S., Kovalenko M., Shevchenko E., Chem. Rev. 2010, 110, 389.

${ }^{2}$ Nag A., Kovalenko M.V., Lee J.S., Liu W., Spokoyny N., Talapin D.V., J Am Chem Soc 2011, 133, 10612.

${ }^{3}$ Koh W.K., Saudari S.R., Fafarman A. T., Kagan C.R., Murray C. B., Nano Lett. 2011, 11, 4764.

${ }^{4}$ Tang J., Kemp K. W., Hoogland S., Jeong K. S., Liu H., Levina L., Furukawa M., Wang X., Debnath R., Cha D., Chou K. W., Fischer A., Amassian A., Asbury J. B., Sargent E. H., Nature Mat. 2011, 10, 765.

${ }^{5}$ Kovalenko, M. V.; Scheele, M.; Talapin, D. V. Science 2009, 324, 1417.

${ }^{6}$ Konstantatos G., Badioli M., Gaudreau L., Osmond J., Bernechea M., Pelayo Garcia de Arquer F., Gatti F., Koppens F. H. L., Nature Nano. 2012, 7, 363.

${ }^{7}$ Kuemmeth F., Bolotin K. I., Shi S.-F., Ralph D. C., Nano Lett. 2008, 8, 4506.

${ }^{8}$ Prins F., Buscema M., Seldenthuis J. S., Etaki S., Buchs G., Barkelid M., Zwiller V., Gao Y., Houtepen A. J., Siebbeles L. D. A., van der Zant H. S. J., Nano Lett. 2012, 12, 5740.

${ }^{9}$ Willis L. J., Faireld J. A., Dadosh T., Fischbein M. D., Drndic M, Nano Lett 2009, 9, 4191.

${ }^{10}$ Yu Q., Cui L., Lequeux N., Zimmers A., Ulysse C., Rebuttini V., Pinna N., Aubin H. ACS Nano 2013, 7, 1487.

${ }^{11}$ Mentzel T.S., Wanger D. D., Ray N., Walker B. J., Strasfeld D., Bawendi M. G., Kastner M. A., Nano Lett. $2012,12,4404$.

${ }^{12}$ Fursina A., Lee S., Sofin R. G. S., Shvets I. V., Natelson D., Appl. Phys. Lett. 2008, 92, 113102.

13 Lin Y.C., Bai J., Huang Y., Nano Lett. 2009, 9, 2234.

${ }^{14}$ Dorn A., Huang H., Bawendi M. G., Nano Lett. 2008, 8, 1347.

${ }^{15}$ Xing W., Kung S.C., van der Veer W. E., Yan W., Ayvazian T., Yun Kim J., Penner R. M., ACS Nano 2012,6, 5627.

${ }^{16}$ Ithurria, S.; Dubertret, B. J. Am. Chem. Soc. 2008, 130, 16504.

17 Ithurria, S.; Tessier, M. D.; Mahler, B.; Lobo, R. P. S. M.; Dubertret, B.; Efros, A. L. Nat. Mater. 2011, 10, 936-941

18 Lhuillier E., Robin A., Ithurria S., Aubin H., Dubertret B., Nano Lett. 2014, 14, 2715.

${ }^{19}$ Dayen J-F, Faramarzi V., Pauly M., Kemp N. T., Barbero M., Pichon B. P., Majjad H, Begin-Colin S, Doudin B., Nanotechnology 2010, 21, 335303.

${ }^{20}$ J.-F. Dayen, E. Devid, M.V. Kamalakar, D. Golubev, C. Guédon, V. Faramarzi, B. Doudin, S.J. van der Molen. Advanced Materials, 2013, 25, 400.

${ }^{21}$ M. Pauly, J.-F. Dayen, D. Golubev, J.-B. Beaufrand, B. Pichon, B. Doudin , S. Bégin-Colin, Small 2012, 8, 108.

22 Ithurria S., Talapin D. V., J. Am. Chem. Soc. 2012, 134, 18585.

${ }^{23}$ The incident power is estimated by the product of the power density by the area of the device.

${ }^{24}$ Lee, J. S.; Kovalenko, M. V.; Huang, J.; Chung, D. S.; Talapin, D. V. Nat. Nanotechnol. 2011, 6, 348.

${ }^{25}$ Konstantatos, G.; Howard, I.; Fischer, A.; Hoogland, S.; Clifford, J.; Klem, E.; Levina, L.; Sargent, E. H. Nature 2006, $442,180$.

${ }^{26}$ Benchamekh R., Gippius N. A., Even J., Nestoklon M. O., Jancu J.-M., Ithurria S., Dubertret B., Efros Al. L., Voisin P., Phys. Rev. B 2014, 89, 035307.

${ }^{27}$ Achtstein A.W., Schliwa A., Prudnikau A., Hardzei M., Artemyev M. V., Thomsen C., Woggon U., Nano Lett. $2012,12,3151$.

${ }^{28}$ Liu H., Lhuillier E., Guyot-Sionnest P., J. Appl. Phys. 2014, 115, 154309.

${ }^{29}$ Lai Y., Li H., Kim D. K., Diroll B. T., Murray C. B, Kagan C. R., ACS Nano 2014, 8, 9664.

${ }^{30}$ Lhuillier E., Pedetti S. , Ithurria S., Heuclin H., Nadal B., Robin A., Patriarche G. , Lequeux N., Dubertret B., ACS nano 2014, 8 , 3813.

${ }^{31}$ Kang, M. S., Lee, J., Norris, D. J., Frisbie, C. D. Nano Lett. 2009, 9, 3848.
} 